\title{
TOWARDS A FRAMEWORK FOR INTEGRATED AND COLLABORATIVE KNOWLEDGE MANAGEMENT FOR ENGINEERING DESIGN - A CASE STUDY
}

\author{
N. Fatfouta ${ }^{1,2, \otimes}$ and J. Stal-Le Cardinal ${ }^{1}$ \\ ${ }^{1}$ CentraleSupélec, France, ${ }^{2}$ Renault, France \\ $\triangle$ naouress.fatfouta@centralesupelec.fr
}

\begin{abstract}
In automotive industry, the design process is costly and time-consuming. Car safety is a crucial factor in the development of a vehicle, which is why crash simulation is an essential step in the design process. To improve car crash simulation analysis, it is necessary to reduce the time required and support the resolution of encountered design issues. We propose a knowledge management approach to support car crash simulation analysis and ensure the collaboration of different stakeholders. In a knowledge-intensive context, we used an ontology-based approach to formalise and capture knowledge.
\end{abstract}

Keywords: knowledge management, collaborative design, car crash simulation, ontology, engineering design

\section{Introduction}

In the automotive industry, the design process is both expensive and time-consuming. This research focuses on improving the design process by reducing time and costs while producing more robust vehicles. At the end of the development phase, the vehicle model must be at the right level of performance and the right manufacturing cost. Vehicle development requires collaboration and integration of the diverse knowledge of different stakeholders and disciplines. Vehicle development is based on modelling and simulation.

Road incidents are ranked ninth among the world's disease burdens in 2016 (Ritchie and Roser, 2018), and they are predicted to rank third by 2020 (Patane et al., 2015). Hence, car safety is a crucial factor for car manufacturers. Crash simulation is an essential step in vehicle development. Therefore, the automotive industry has the widest application of such simulation (Yadav and Pradhan, 2014). Crash simulation and more generally engineering analysis is knowledge intensive; it requires comprehensive expert knowledge. Within nowadays organisations, engineers and experts change missions and teams very often. Knowledge is usually transferred via communication. Thus, knowledge management is playing a crucial role in today's industry. Knowledge management refers to identifying and leveraging collective knowledge (Alavi and Leidner, 2001; Barão et al., 2017). It aims to reuse knowledge through transferring it into information and between participants (Peng et al., 2017).

Therefore, this paper aims to propose a knowledge management approach to support the development phase of vehicles, mainly car crash simulation.

This article is organised as follows. Section 2 presents a literature review on knowledge management in engineering design. Then, section 3 explains the case study. Section 4 proposes an integrated and 
collaborative knowledge management framework with a focus on the knowledge model and knowledge retrieval model. Finally, conclusions and outlook are given in section 5.

\section{Literature review: Knowledge management in engineering design}

Design involves people with the appropriate expertise undertaking a process to develop a product; This takes place within an organisation that provides necessary infrastructure and resources (Wallace et al., 2005). Design tasks, including within the automotive industry, are now more complex, their timescales are increasing and their teams are geographically distributed (Mcmahon et al., 2004). Engineering design process is knowledge-intensive and collaborative (Peng et al., 2017). It requires multiple designers and experts to conduct multiple tasks involving different areas of knowledge and expertise (Sun et al., 2010). Collaborative engineering design is evolving towards a problem-solving task (Chen et al., 2008) that embodies a significant level of complexity. Engineering design is heavily informational; engineering designers spend considerable time locating information in human and nonhuman sources (Peng et al., 2017; Sun et al., 2010); they engage 55.75\% of their time in informational behaviour, such as processing, communicating and disseminating information (Robinson, 2010). Human sources are more solicited than non-human ones (Robinson, 2010). However, due to the current transient nature of industrial organisations, experienced designers and experts are not going to be available to consult in the future (Wallace et al., 2005). The multidisciplinary, highly collaborative and contextual nature of engineering design has raised the need to support integrated and collaborative product development (Peng et al., 2017). Successful collaborative engineering design depends on the ability to manage and share engineering knowledge (Chen et al., 2008). Therefore, nowadays, knowledge management is playing a crucial role for companies' competitiveness (Barão et al., 2017).

There are multiple definitions of knowledge management (KM) in literature (Girard et al., 2015). It is usually defined by its relationship with knowledge. Therefore, we start by defining knowledge.

Many ways of defining knowledge exist in literature. Knowledge is usually defined using a hierarchical relationship with data and information (Alavi and Leidner, 2001; Peng et al., 2017; Sun et al., 2010): where (1) data is in the form of symbols and words, (2) information is structured data that forms a pattern, and (3) knowledge is processed information and rules of thumb acquired through experience. Knowledge can also refer to a state of knowing, the "know-about", a capacity for action, the "know-how", and to articulated and captured facts and methods, the "body of knowledge" (Apurva and M.D., 2011). Another frequent dichotomy of knowledge is based on its stage of accessibility: explicit, implicit and tacit (Apurva and M.D., 2011; Mcmahon et al., 2004; Sun et al., 2010). Explicit knowledge, also identified as formal knowledge (Peng et al., 2017), is knowledge that can be codified and documented. Implicit knowledge can be implied by or inferred from observable behaviour or performance. Tacit knowledge is subconscious and cannot be expressed. In the context of engineering knowledge, Wallace et al. (Wallace et al., 2005) classify engineering design knowledge into product and process knowledge. When it is stored externally, it is considered as information and can either describe the design process or the product itself. When it is sored un human memory, knowledge can be explicit, implicit and tacit. Explicit knowledge is an explanation of both the process and the product and can be articulated. Implicit knowledge is the understanding the engineers have of both process and product and cannot be expressed by engineers themselves; it can be articulated through knowledge elicitation. Tacit knowledge is more a matter of intuition and cannot be articulated.

Given the different definitions and perspectives of knowledge, knowledge management (KM) has multiples definitions. KM refers to identifying and leveraging collective knowledge (Alavi and Leidner, 2001; Barão et al., 2017) and it is usually defined by its processes including knowledge capture, storing, retrieval and transfer (Al-Emran et al., 2018; Alavi and Leidner, 2001; Girard et al., 2015). KM is an essential asset in modern institutions; it enables learning from corporate memory, growth, success and innovation (Al-Emran et al., 2018). In engineering design, knowledge can be in form of tangible objects or experience learnt and accumulated through a community of expertise; this constitute both approaches of KM, the codification and the personalisation view (Peng et al., 2017). Most common approaches are technology-oriented, called codification approach; it underlines the explicit nature of knowledge that can be formalised and stored in knowledge repositories and transferred via information and communication technologies. Then, people-oriented also called 
personalisation approach underlines the tacit and context-dependent nature of knowledge; it requires informal human communication to transfer knowledge (Peng et al., 2017; Saito et al., 2007). It is argued that a trade-off between the two approaches, called integrated approach, is more effective ( $\mathrm{Ng}$ et al., 2012; Peng et al., 2017; Saito et al., 2007). An integrated approach to KM can improve the quality and efficiency of design through the capture and reuse of informal and contextual knowledge.

\section{Case study: Car crash simulation}

This research is conducted within a French multinational automotive company. Within this company, the development phase is a succession of design analysis loops, terminating in milestones. At the end of the development phase, the vehicle model must be at the right level of performance and the right manufacturing cost. The development phase consists of iteratively refining the design specifications, evaluating the vehicle's performance and solving design issues encountered. This phase is supported by modelling and simulation. We are in a context of simulation-aided design as decision about the vehicle design is mainly based on simulation. Several variations on a vehicle model exist at the same time, as there are several markets with different expectations.

The development phase consists of different processes. The design process consists of creating and updating the design reference representing the knowledge gathered about the vehicle under development. The customer performance specification process provides the specification of the vehicle. The simulation model generation process consists of creating and updating the digital vehicle models required for the simulation. The simulation process evaluates the vehicle's performance and solves the design issues revealed; a design issue occurs when the performance does not meet the specification. Finally, the decision-making process determines which design changes to incorporate. A design change is a solution to the design issue. Different stakeholders are involved in these processes.

According to an empirical study we conducted within the company (Fatfouta et al., 2019), car crash simulation is expensive, time-consuming, and there is considerable effort required. Therefore, we focus on crash simulation and mainly on the analysts involved. The analysts' mission is to deliver a vehicle model compliant with multiple requirements. They rely on simulation to evaluate vehicle performance. Figure 1 presents a formalisation of the simulation process including design issue resolution and design change proposals. During the development phase, for a design analysis loop, crash simulation analysts receive the specification and the vehicle model to evaluate. Following the simulation results, they proceed with the interpretation of these results. If the model fails to meet specification, a design issue is identified. The analysts assess and diagnose the design issue, then propose design changes to fix it. If the design change is not good enough, they consider a new path for another design change. If the design change satisfies the requirement, the project decision-makers decide whether to implement it in the model. This decision depends on project factors including cost and weight. The decision-making process is not considered in this research; only the resulting decision is considered. At the end of each design analysis loop, all design changes that have been approved by the different disciplines, such as crash and stress, are incorporated into the vehicle model, ready for the next loop.

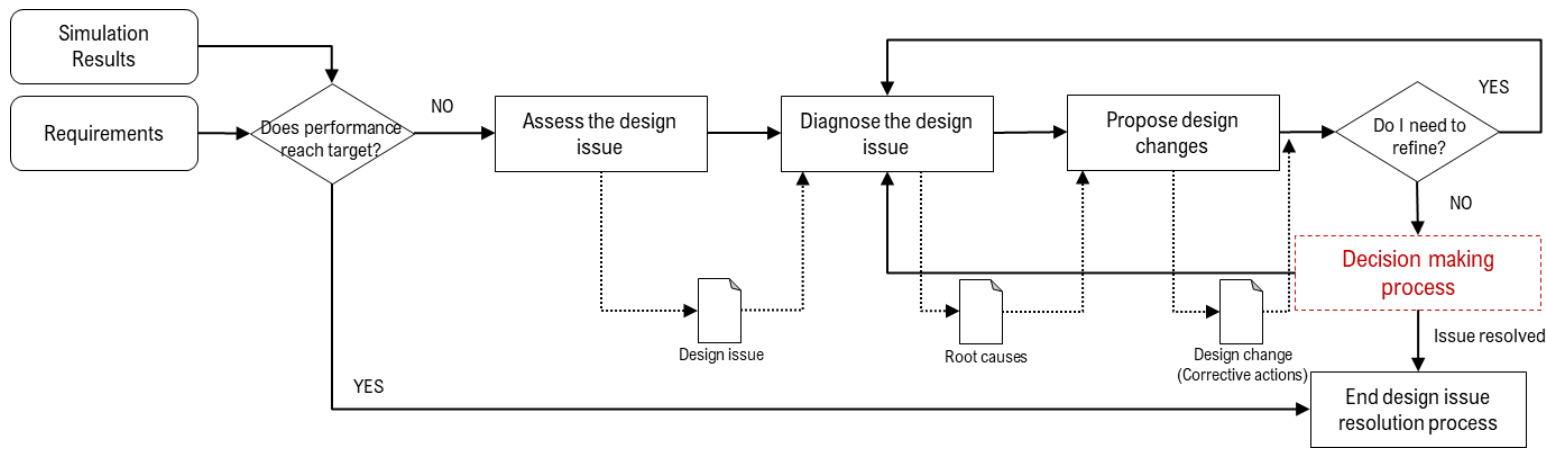

Figure 1. Design-issue resolution and design-change proposal formulation

Analysts are required to have knowledge of modelling and simulation. They are also required to make decisions on paths for addressing design issues and proposing design changes. Car crash simulation 
analysts are geographically decentralised, and there are only one crash simulation expert and one digital simulation expert in the company (Fatfouta et al., 2019). Car crash simulation analysts collaborate with different stakeholders. They collaborate with other crash simulation analysts from other work sites and with simulation analysts from different disciplines such as stress simulation analysts. A strong collaboration exists with the simulation experts as they have the most significant expertise within the company. Analysts, designers and customer-performance specialists exchange information on the statues of the resolution of design issues. Finally, decision-makers are involved in decision-making process regarding the design changes to be incorporated into models.

Simulation analysts encounter several challenges within car crash simulation (Fatfouta et al., 2019). They need to quickly locate all relevant data and knowledge, from different sources, spending as little time as possible. Design-issue analysis and design-change proposal formulation need to be supported, in order to reduce the time spent on simulation and reduce bias of less experienced analysts when interpreting simulation results.

\section{Framework of integrated and collaborative knowledge management}

In this research, we perform participation action research (PAR). Being part of the organisation under study allows us to contribute to increasing the knowledge that can be used by the practitioner, and being part of a scientific environment enables us to contribute to science (Björk and Ottosson, 2007).

The aim of this paper is to improve simulation-aided design; time needs to be reduced and decisions about investigation paths and design change proposals must be substantiated. Crash simulation is knowledge intensive and depends heavily on expertise. Since there is only one crash simulation expert, it is important to capture the knowledge and expertise, knowing the risk that the expert may not be available. Collaboration between the different participants is very important for the successful running of the design process. Taking these characteristics into consideration, we propose a knowledge management approach to improve simulation-aided design through the support of analysts and collaboration with the different stakeholders involved.

To develop our proposal, we conducted an extensive literature review on existent knowledge management frameworks (Alavi and Leidner, 2001; Chen et al., 2008, 2012; Li et al., 2009; Pawlowski and Bick, 2012; Peng et al., 2017), while considering the specifics of our industrial context mentioned above. In previous work (Fatfouta et al., 2019), we proposed formalising knowledge related to car crash simulation using an ontology. The interest in developing ontologies is growing in engineering design as it involves knowledge sharing and the development of a common standard language for the formalisation of domain knowledge (Premkumar et al., 2014). An ontology is an explicit specification of a shared conceptualisation, and any knowledge base or knowledge-based system is, implicitly or explicitly, linked to conceptualisation (Iaksch and Borsato, 2019; Kestel et al., 2019). Thus, the literature review was performed in light of this ontology. To our knowledge, there is no ontology-based knowledge management approach to support simulation-aided design, for the development phase of vehicles. Moreover, since we perform PAR, the proposal is formalised and discussed with practitioners, including engineers responsible for the development of this support, experts and end-users.

\subsection{Ontology-based knowledge management}

The ontology we have developed in previous work is called the Crash Simulation Post-Processing Ontology (Fatfouta et al., 2019). This ontology formalises knowledge related the post-processing phase of car crash simulation and the interpretation of simulation results. The aim is to formalise knowledge related to the resolution of design issues and the proposal of design changes. To better structure engineering knowledge, the ontology is formalised in three levels: the context level, the project level and the reasoning level:

- The context level describes information about the context in which the digital test happens such as the requirements to meet and the impact configuration, such as a frontal and a rear crash.

- The project level formalises engineering knowledge related to the vehicle project. We considered all necessary elements to ensure an accurate interpretation of simulation results. 
- The reasoning level formalises the resolution of design issues and the formulation of design change proposals. The reasoning level is independent of the project.

In this paper, for brevity, we focus on the reasoning level of the ontology as it represents the engineering knowledge related to the resolution of design issues. Currently, analysts solve design issues and propose design changes based on their implicit and tacit knowledge and expertise. The reasoning level intends to capture and formalise the reasoning behind the resolution of design issues. It would also help to classify the separate cases into overall categories.

As the reasoning level is independent of the project, we deal with a generic vehicle model. The instantiation of the generic vehicle model is a project vehicle model for a specific context. A generic vehicle model is composed of generic vehicle parts, just as a project vehicle model is composed of vehicle parts. Each vehicle part has a specific role to play, within the vehicle structure, during the crash, to ensure the safety of the occupants. As explained in Figure 2, A Generic Vehicle Part is defined by its Role to play and an expected Behaviour to ensure that role. Then, a Generic Issue exists if the Generic Vehicle Part does not play well its Role, and it engages a defect Behaviour. Thus, a Generic Issue is defined as a Behaviour-Gap between the expected Behaviour and the defect Behaviour of the considered Generic Vehicle Part. A Generic Issue has a Generic Root Cause. Once the Generic Root Cause is identified, a proposal of a Generic Corrective Action is done. A Generic Corrective Action is composed of Elementary Actions, such as Material Change, Geometric Change and Design of a new part. A design change is one or multiple corrective actions.

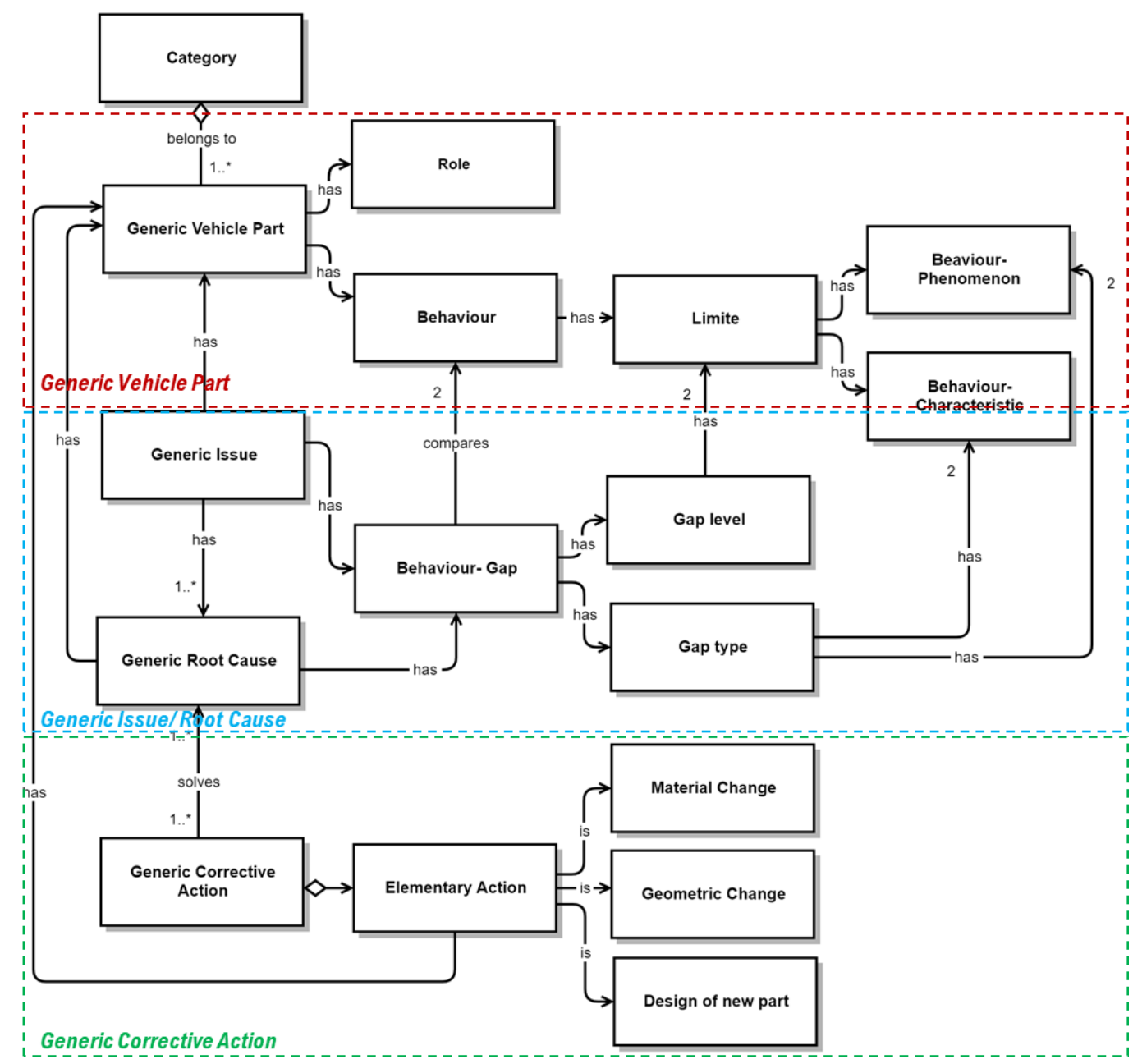

Figure 2. The reasoning level of the ontology: Focus on the design-issue resolution

The abstraction of the reasoning level is based on two hypotheses. First, crash simulation is based on physical and mechanical phenomena, so the behaviour of vehicle parts has limited deformation 
possibilities and can be predicted. Second, vehicle parts sharing the same role and behaviour would be exposed to similar issues. Generic vehicle parts with similar roles and behaviours would share similar generic issue and could belong to the same Category. Therefore, design issues with similar diagnoses (root causes) would require similar design changes.

\subsection{A framework of a collaborative and integrated knowledge management}

In this paper, we propose a framework of an integrated and collaborative knowledge management frameworks which integrate the developed ontology as a knowledge model. This framework aims to support simulation-aided design. Figure 3 shows the proposal of the KM framework. KM processes are presented, starting from knowledge capture. Engineering knowledge will be captured as the project proceeds. Three type of engineering knowledge are identified. First, there is information about vehicle projects, such as vehicle models and digital tests. Then there is explicit knowledge, including knowledge related to the assessment of design issues, root causes and design changes. Finally, implicit knowledge relates to the paths of investigation and the know-how associated with proposed design changes.

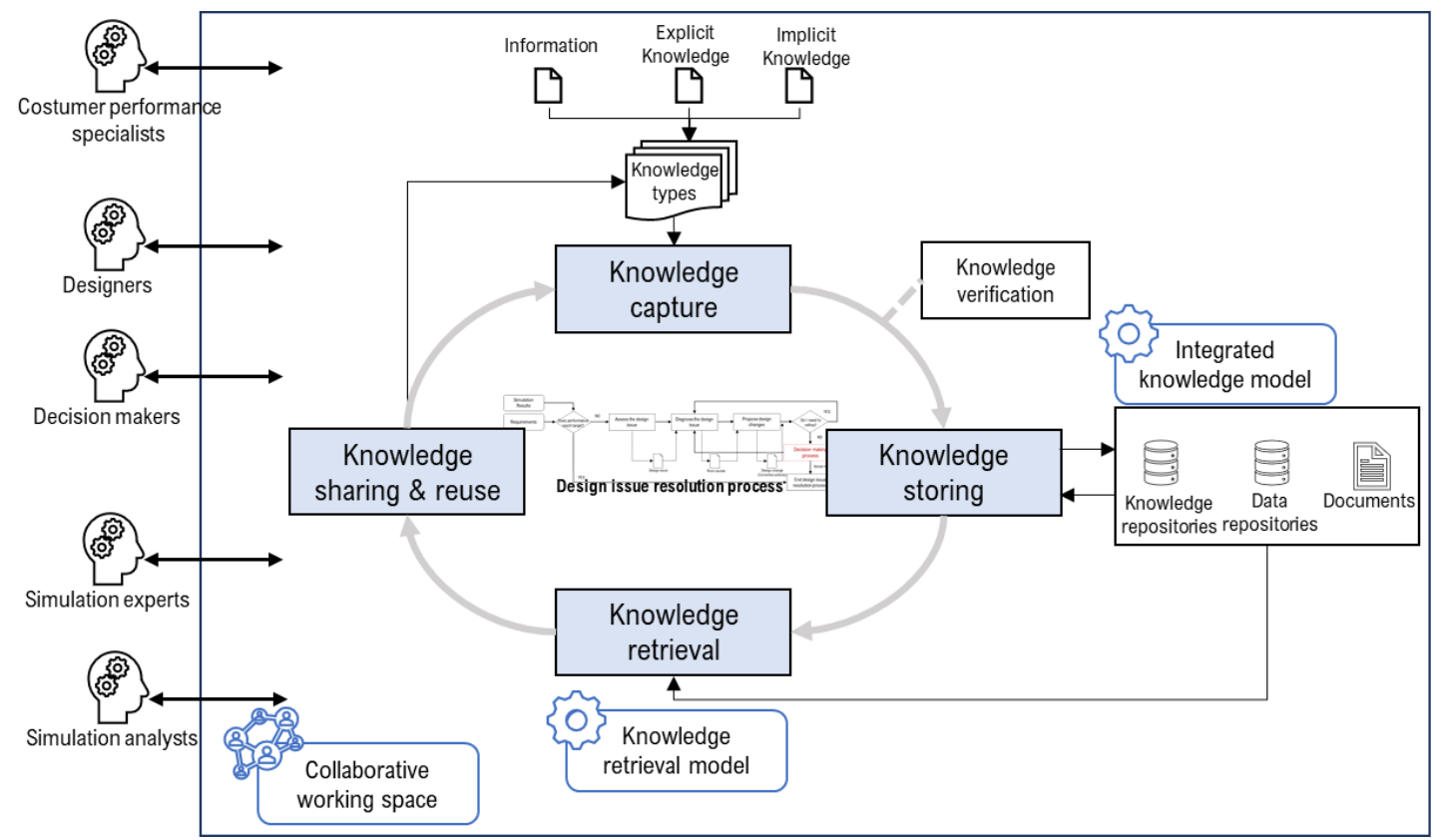

Figure 3. A proposal of a framework of a virtual integrated and collaborative knowledge management

Before storing captured knowledge, it must be verified and validated. This could be done by knowledge workers, such as experts. Knowledge is stored according to the ontology, an integrated knowledge model. The repositories retain engineering knowledge including evaluation of simulation results, design issues and design changes. They also store contextual information, such as vehicle models, and specifications. Documents and discussions between participants can also be stored, as they may contain comprehensive information that could help other participants better understand. The KM framework is then integrated since it is based on an integrated knowledge model that captures both information and formal knowledge and considers the implicit and context-dependent nature of knowledge. In addition, the knowledge is retrieved by a search engine based on a knowledge search model. When retrieved, the knowledge is shared and reused. Hence, knowledge is enhanced, created and re-captured.

Supporting collaborative design is important for this framework and it ensured by the collaborative working space. Collaboration and communication can take place between different participants such as analysts, designers and experts. Communication has an important role to play in KM to facilitate knowledge sharing and reuse (Peng et al., 2017). It is therefore necessary to ensure it throughout the project. Identifying the experience and expertise of participants is also important, as it would make it easy to identify the contact person if needed. Decision-makers are also considered; this would give more visibility on the decision-making process. 


\subsection{Knowledge management system architecture}

A knowledge management system (KMS) is an information system applied to manage corporate knowledge and is developed to support and improve $\mathrm{KM}$ processes in organisational workplaces (Barão et al., 2017). The KMS aims to support engineering collaborative activities. It would supply engineers with contextual and accurate knowledge, and it would also ensure knowledge capture and reuse throughout the collaborative process. The KM support system is expected to significantly reduce the crash simulation time by reducing the time spent on design-issue resolution and design-change proposal. It would also increase the accuracy of proposals for more efficient design changes. Our proposal is based on an extended literature review of the KMS architecture (Desta et al., 2014; Li et al., 2009; Peng et al., 2017; Sun et al., 2009; Zhang et al., 2011). The main requirements of the proposed KMS are to support knowledge workers in their activities within the collaborative process and to enable the transfer and sharing of knowledge between individuals and teams.

The system architecture is explained in Figure 4 and consists of four main parts: three layers and a multi-user interface (MUI). The MUI ensures the performance of KM activities and interaction between the users within the integrated working environment. $\mathrm{KM}$ is also distributed as it offers the users or a group of them distributed working spaces for individual and collaborative tasks within the teams. The three layers consists of resources layer, methodologies layer and models layer.

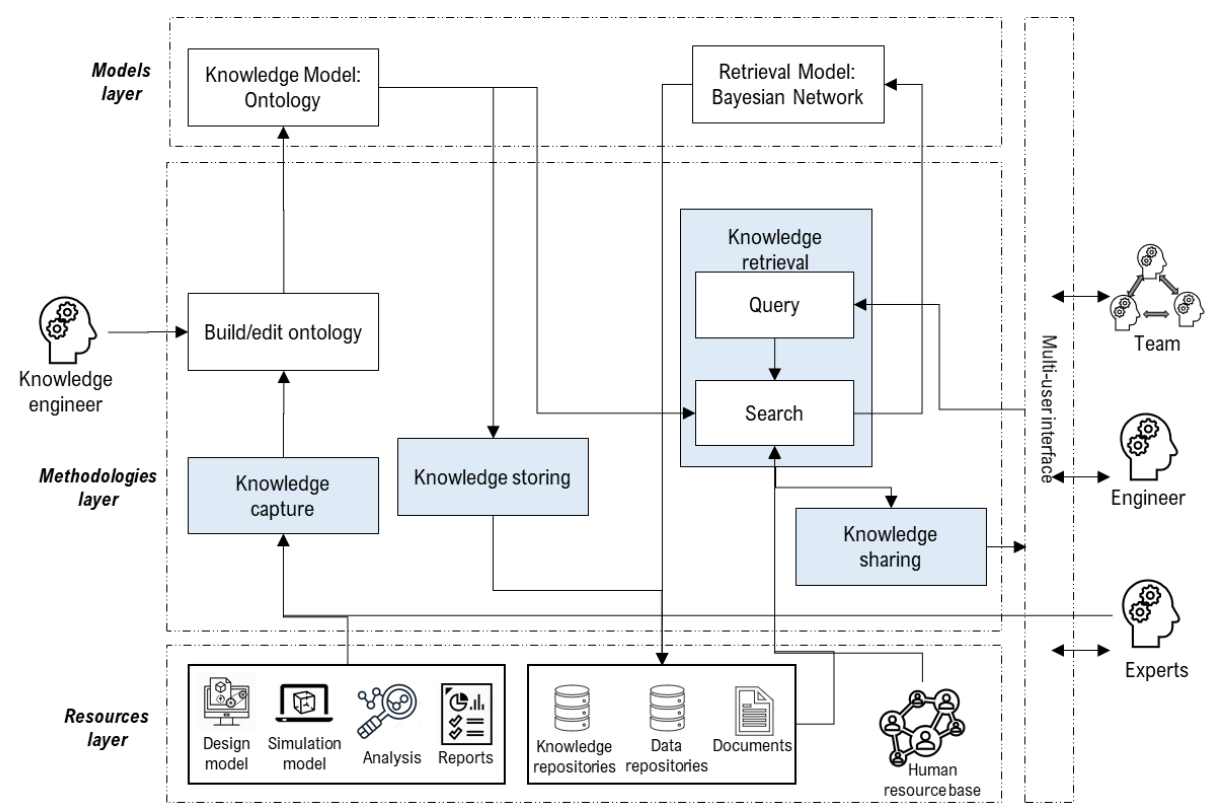

Figure 4. A proposal of a knowledge management system architecture

- The resources layer refers to knowledge repositories in various data and knowledge formats. The repositories include captured information and knowledge as explained above. Implicit and tacit knowledge embedded in the human mind could be shared through communication. Therefore, the human resource base of each employee's roles, skills and abilities, is available.

- The models layer consists of models developed to support the implementation of the system including the integrated knowledge model and the knowledge retrieval model. The integrated knowledge model is the Crash Simulation Post-Processing Ontology. The retrieval model will be based on a Bayesian network and will be subject to future work.

- The methodologies layer represents the methodologies used to carry out KM activities. Some of the main connections are explicated (in the Figure 4). For example, knowledge can be captured from experts and from formal knowledge elements. To store knowledge, the knowledge engineer must build/edit the ontology. Knowledge retrieval is usually accessible via the MUI. The user expresses his query, then the search uses the retrieval model. Depending on the query, the search accesses the ontology, the knowledge repositories and the human source base. Finally, knowledge is shared and transferred via the MUI. 


\subsection{Use case scenario}

As an application of the proposed KMS, we propose to explain a use case scenario based on 3 cases detailed in Figure 5. These cases represent 3 different design issues and their design changes, encountered in different projects, for different vehicle models, solved and documented by different analysts, but occurred for the same impact configuration (Rear RCAR).

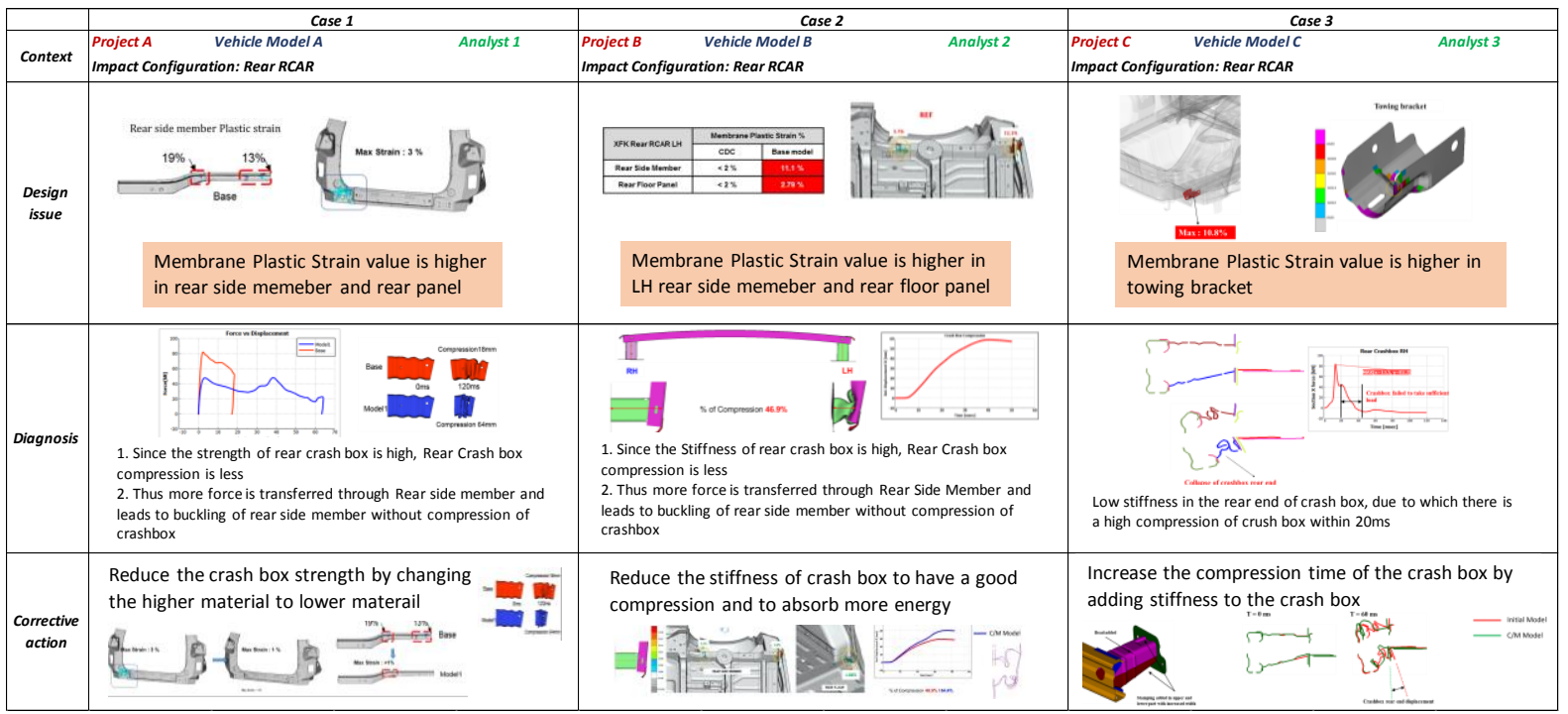

Figure 5. Three cases of actual design-issue resolution used for the use case scenario

To better understand the KMS use case scenario, we begin by explaining the role of ontology and the reasoning that will be used in the knowledge retrieval. Figure 6 presents a brief instantiation of the reasoning level ontology for case 1 . In case 1, the encountered design issue is that membrane plastic strain (MPS) of the side member and the rear panel is too high. The objective is to identify the elementary root cause. An elementary root cause is a cause for which analysts can propose corrective actions. Thus, for the first iteration, the root cause identified is the low compression of the crash box. This cause is not elementary because the analyst cannot propose a design change to address it. Thus, for the second iteration, the root cause of the crash box's low compression is its high stiffness. High stiffness is elementary. Therefore, the corrective action is to reduce stiffness. In this case, the analyst proposes to reduce it by changing materials.

\begin{tabular}{|c|c|c|c|c|c|c|c|c|c|c|c|}
\hline \multirow{3}{*}{ Iteration } & \multirow{3}{*}{$\begin{array}{l}\text { Vehicle } \\
\text { part }\end{array}$} & \multirow{3}{*}{ Role } & \multirow{3}{*}{$\begin{array}{c}\text { Expected } \\
\text { Behaviour }\end{array}$} & \multicolumn{3}{|c|}{ Issue } & \multicolumn{4}{|c|}{ Root Cause } & \multirow{3}{*}{$\begin{array}{c}\text { Corrective } \\
\text { action }\end{array}$} \\
\hline & & & & \multirow{2}{*}{$\begin{array}{c}\text { Defect } \\
\text { behaviour }\end{array}$} & \multicolumn{2}{|c|}{ Behaviour Gap } & \multirow{2}{*}{$\begin{array}{c}\text { Defect } \\
\text { behaviour }\end{array}$} & \multicolumn{2}{|c|}{ Behaviour Gap } & \multirow{2}{*}{ Elementary } & \\
\hline & & & & & Gap level & Gap Type & & Gap level & Gap Type & & \\
\hline & $\begin{array}{c}\text { Side } \\
\text { memeber }\end{array}$ & $\begin{array}{l}\text { Absorb } \\
\text { energy }\end{array}$ & $\begin{array}{l}\text { MPS within a } \\
\quad \text { limit } \mathrm{L}\end{array}$ & MPS $>$ L & $\mathrm{X}$ & & & & & & \\
\hline $\begin{array}{c}\text { Iteration } \\
\quad 1\end{array}$ & Crash Box & $\begin{array}{l}\text { Absorb } \\
\text { energy }\end{array}$ & $\begin{array}{c}\text { compress within } \\
\text { a limit L1 }\end{array}$ & $\begin{array}{c}\text { Compression } \\
<\mathrm{L} 1\end{array}$ & $\mathrm{X}$ & & $\begin{array}{c}\text { Compression } \\
<\mathrm{L} 1\end{array}$ & $\mathrm{X}$ & & & \\
\hline $\begin{array}{c}\text { iteration } \\
2\end{array}$ & Crash Box & $\begin{array}{l}\text { Absorb } \\
\text { energy }\end{array}$ & stiffness $\mathrm{S}$ & & & & Stiffness $>\mathrm{S}$ & $\mathrm{X}$ & & $\mathrm{X}$ & $\begin{array}{l}\text { reduce } \\
\text { stiffness }\end{array}$ \\
\hline
\end{tabular}

Figure 6 . Instantiation of the ontology's reasoning level for case 1

In a scenario where the KMS described above is implemented, users interact with it via the MUI. They formulate the query. Based on the query, the search engine will look for similar cases and get the matching results. This is where the Bayesian network intervenes. The network is supposed to link an issue, knowing the context, to its probable causes in a hierarchical way, like the iterations presented in Figure 6 . Then, the elementary root cause will be linked to possible corrective actions. Thereby, the KMS will support analysts throughout the design issue resolution process. Finally, the knowledge will be transferred to the user via the MUI and re-captured to improve the existing knowledge in the repositories. 
Based on the assumption that case 1 is already captured via the ontology and stored within knowledge repositories, we explain briefly use case scenario of the support provided by the KMS for cases 2 and 3 (from Figure 5). In case 2, the analyst faces a similar design issue in a context with some similarities. Thus, the knowledge retrieval will most probably make it possible to extract case 1 and propose to check the stiffness of the crash box. For case 2, according to the analysis, the stiffness of the crash box was also high, and the corrective action will then be to reduce it. The corrective action is evaluated and approved, so the analyst can formulate the design change proposal. In case 3, for a similar design issue, the root cause is also related to the stiffness of the crash box but in this case, it is rather low. In this case, based on the implicit knowledge captured, the system would propose to increase stiffness. If the search does not find a match to the design issue, or if the request is to identify an expert or engineer, the search will be conducted in the human source base. It would suggest the most appropriate expert/ engineer to meet the request. Communication would be provided by the MUI. The interaction would be recorded. Then, if necessary, analysts would have access to it to formulate their own judgements and draw lessons.

\section{Conclusions and outlook}

Car crash simulation analysis is characterised by its knowledge intensive nature and human-based interactions. More precisely, it relies heavily on experts. These characteristics can be a source of inefficiency in the overall engineering process due to the unavailability of experts and the long time needed for information retrieval. To address these issues, we propose, in this paper, an ontology-based KM framework and a KMS architecture to support simulation-aided design, with a focus on crash simulation.

The KMS will capture and retain engineering knowledge, including knowledge related to the evaluation of simulation results, assessment of design issues and design change proposals. It will also ensure collaboration. The knowledge management support system would help reduce crash simulation time by reducing the time spent solving design issues and proposing design changes, as well as the time spent searching for a human source of information. This would also increase the accuracy of proposals for more effective design changes, since the proposals will be based on corporate knowledge. This could lead to the development of more robust vehicle models. Novice analysts would have access to the engineering knowledge retained by the KMS, making it a learning tool.

Future work will focus on exploring knowledge retrieval and the applicability of Bayesian networks. The framework could be extended to the simulation of all performances, such as noise and vibration. It could also include the design process, thus avoiding that certain design issues arise during the simulation and avoiding proposing design changes that cannot be included in the vehicle model (design). The framework could also go beyond simple collaboration to real-time concurrent engineering where each participant in each discipline could access the same vehicle model at the same time.

\section{References}

Al-Emran, M. et al. (2018), "The impact of knowledge management processes on information systems: A systematic review”, International Journal of Information Management, Elsevier Ltd, 1 December.

Alavi, M. and Leidner, D.E. (2001), "Review: Knowledge Management and Knowledge Management Systems: Conceptual Foundations and Research Issues", Source: MIS Quarterly, Vol. 25.

Apurva, A. and S, M.D. (2011), "Understanding Knowledge Management: a literature review", International Journal of Engineering Science and Technology (IJEST), Meyer \& Zack, Vol. 3 No. 2, pp. 926-939.

Barão, A. et al. (2017), "A knowledge management approach to capture organizational learning networks", International Journal of Information Management, Elsevier Ltd, Vol. 37 No. 6, pp. 735-740.

Björk, E. and Ottosson, S. (2007), "Aspects of consideration in product development research", Journal of Engineering Design, Vol. 18 No. 3, pp. 195-207.

Chen, Y.J., Chen, Y.M. and Chu, H.C. (2008), "Enabling collaborative product design through distributed engineering knowledge management", Computers in Industry, Vol. 59 No. 4, pp. 395-409.

Chen, Y.J., Chen, Y.M. and Wu, M.S. (2012), "An empirical knowledge management framework for professional virtual community in knowledge-intensive service industries", Expert Systems with Applications, Vol. 39 No. 18, pp. 13135-13147. 
Desta, T., Garfield, M. and Meshesha, M. (2014), Proposing a Knowledge Management System (Kms) Architecture to Promote Knowledge Sharing Among Employees, available at: http://aisel.aisnet.org/ecis2014http://aisel. aisnet.org/ecis2014/proceedings/track04/7 (accessed 11 November 2019).

Fatfouta, N. et al. (2019), "An ontology towards a knowledge-based support of the post-processing phase of car crash simulation”, Advanced Engineering Informatics, Vol. submitted.

Fatfouta, N., Stal-Le Cardinal, J. and Royer, C. (2019), "Empirical Study of Car Crash Simulation Analysis within the Development Phase", 22nd International Conference on Engineering Design ICED, Delft, The Netherlands, pp. 2843-2852.

Girard, J., Georgia, M. and College, S. (2015), "Defining knowledge management: Toward an applied compendium", Online Journal of Applied Knowledge Management, Vol. 3 No. 1, pp. 1-20.

Iaksch, J.S. and Borsato, M. (2019), "Method for digital evaluation of existing production systems adequacy to changes in product engineering in the context of the automotive industry", Advanced Engineering Informatics, Vol. 42, p. 100942.

Kestel, P. et al. (2019), "Ontology-based approach for the provision of simulation knowledge acquired by Data and Text Mining processes", Advanced Engineering Informatics, Elsevier, Vol. 39 No. December 2018, pp. $292-305$.

$\mathrm{Li}, \mathrm{H}$. et al. (2009), "A framework of ontology-based knowledge management system", Proceedings - 2009 2nd IEEE International Conference on Computer Science and Information Technology, ICCSIT 2009, pp. 374-377.

Mcmahon, C., Lowe, A. and Culley, S. (2004), "Knowledge management in engineering design: personalization and codification", Journal of Engineering Design, Vol. 15 No. 4, pp. 307-325.

Ng, A.H.H. et al. (2012), "Integrated Knowledge Management Strategy: A Preliminary Literature Review”, Procedia Social and Behavioral Sciences, Elsevier BV, Vol. 57, pp. 209-214.

Patane, S., Narkar, K.M. and Panchagade, D.R. (2015), "Evaluation of Tools \& Techniques used for Frontal Crash Analysis", available at: https://www.semanticscholar.org/paper/Evaluation-of-Tools-\%26-Techniques-usedfor-Frontal-Patane-Narkar/72e691342e29110309774c6441494ece375c8074 (accessed 12 September 2019).

Pawlowski, J. and Bick, M. (2012), "The Global Knowledge Management Framework: Towards a Theory for Knowledge Management in Globally Distributed Settings", Journal of Knowledge Management, Vol. 10 No. 1, pp. 92-108.

Peng, G. et al. (2017), "A collaborative system for capturing and reusing in-context design knowledge with an integrated representation model”, Advanced Engineering Informatics, Elsevier, Vol. 33, pp. 314-329.

Premkumar, V. et al. (2014), "A semantic knowledge management system for laminated composites", Advanced Engineering Informatics, Elsevier Ltd, Vol. 28 No. 1, pp. 91-101.

Ritchie, H. and Roser, M. (2018), "Causes of Death", OurWorldInData.Org, available at: https://ourworldindata. org/causes-of-death\#causes-of-death-in-recent-decades.

Robinson, M.A. (2010), “An empirical analysis of engineers' information behaviors", Journal of the American Society for Information Science and Technology, Vol. 61 No. 4, pp. 640-658.

Saito, A., Umemoto, K. and Ikeda, M. (2007), "A strategy-based ontology of knowledge management technologies”, Journal of Knowledge Management, Vol. 11 No. 1, pp. 97-114.

Sun, W. et al. (2010), "Knowledge-intensive support for product design with an ontology-based approach", Int $J$ Adv Manuf Technol, Vol. 48, pp. 421-434.

Sun, W., Ma, Q. and Chen, S. (2009), "A framework for automated finite element analysis with an ontologybased approach", Journal of Mechanical Science and Technology, Vol. 23 No. 12, pp. 3209-3220.

Wallace, K., Ahmed, S. and Bracewell, R. (2005), "Engineering knowledge management", Design Process Improvement, Springer London, London, pp. 326-343.

Yadav, S. and Pradhan, S.K. (2014), "Investigations into Dynamic Response of Automobile Components during Crash Simulation”, Procedia Engineering, Elsevier, Vol. 97, pp. 1254-1264.

Zhang, J. et al. (2011), "Ontology-based knowledge management system and application", Procedia Engineering, Vol. 15, pp. 1021-1029. 